\title{
Inserção no mundo do trabalho odontológico: percepção de graduandos em Odontologia sobre habilidades adquiridas e expectativas
}

\author{
Taiomara Vieira Mania*; Andrea Maria Duarte Vargas**; Efigênia Ferreira e Ferreira**
}

* Mestranda, Departamento de Odontologia Social e Preventiva, Faculdade de Odontologia, Universidade Federal de Minas Gerais

** Professora doutora, Departamento de Odontologia Social e Preventiva, Faculdade de Odontologia, Universidade Federal de Minas Gerais

Recebido em 16/11/2017. Aprovado em 28/07/2018.

\begin{abstract}
RESUMO
Esta pesquisa teve como objetivo avaliar a autopercepção do estudante de odontologia quanto às habilidades adquiridas na graduação para o início de suas atividades profissionais, além de identificar aspectos de seu projeto profissional. Os estudantes do décimo período de duas Instituições de Ensino Superior responderam a um questionário autoaplicável, com perguntas relacionadas às habilidades e competências previstas nas Diretrizes Curriculares Nacionais de Odontologia, e relativas ao seu projeto inicial de vida profissional. Dos 132 questionários distribuídos, 103 foram respondidos. A maioria dos estudantes considerou iniciar carreira em clínica privada e/ou pública $(87 ; 84,46 \%)$, como prestador de serviços. Planejam continuidade de estudos em cursos de especialização e ou aperfeiçoamento (72; 69,89\%); têm segurança em habilidades clínicas relacionadas a procedimentos de Atenção Básica (>70\%) e bem menos em procedimentos especializados. Quanto às habilidades gerais apresentam dificuldades em termos de liderança e gerenciamento $(<42,71)$. Não são fluentes em língua estrangeira $(28,15 \%$,$) e apresentam expectativas de ganhos financeiros próximas à$ realidade. Compreendem bem o mundo do trabalho, percebem a necessidade de continuar os estudos, estão seguros de algumas habilidades gerais e clínicas adquiridas e percebem que algumas estão insuficientes. Rever o modo de ensinar, a relação ensino-aprendizagem e a metodologia de avaliação pode ser um caminho para tornar vivas as Diretrizes Nacionais Curriculares para a Odontologia.

Descritores: Estudantes de Odontologia. Competência Profissional. Ensino. Setor de Assistência à Saúde.
\end{abstract}

\section{INTRODUÇÃO}

Os primeiros passos do ensino superior da Odontologia foram marcados por abordagem tecnicista e organicista, identificada com o modelo biomédico de atenção à saúde. Barros ${ }^{1}$ explica que o alvo do interesse nesse modelo passou da história da doença para uma descrição clínica dos achados propiciados pela patologia, valorizando o aspecto individual sobre o coletivo.

A prática odontológica recebeu influência 
daquele modelo e o ensino era direcionado para o consultório particular e venda de serviços no campo privado. Houve, então, a formação de um profissional restrito a conhecimentos e práticas fragmentadas tendendo para especializações precoces, com baixo alcance populacional ${ }^{2}$.

Entre os anos de 1960 a 1980 ocorreu a expansão da Odontologia. Diante daquele cenário, criaram-se mecanismos de concorrência na busca de se equacionar oferta e procura por serviços; estes movimentos levaram os serviços odontológicos ao culto à especialização como forma de diferenciação profissional e justificativa de custos para o serviço. Se por um lado o mecanismo foi positivo, ao impulsionar o profissional à busca pelo conhecimento, por outro, o aumento do número de profissionais no mercado somado à conjuntura econômico-social iniciou um processo no qual os profissionais passaram a praticar valores mínimos pelos serviços prestados. Flagrou-se uma retração do mercado de trabalho ${ }^{3}$.

Uma das mudanças paradigmáticas que vêm ocorrendo como resposta à situação encontrada na Odontologia consiste na mudança da abordagem do ensino. No Brasil, tendo em vista as novas necessidades da prática odontológica, em 2002, o Conselho Nacional de Educação definiu as Diretrizes Curriculares Nacionais (DCN) para os cursos de graduação em Odontologia. O documento aponta habilidades e competências gerais necessárias para o exercício da profissão como o compromisso com a saúde e a atualização, com a ética e a cidadania, além do desenvolvimento da capacidade de liderança, gerenciamento e comunicação. Tudo isso em um processo que considera a integração curricular como estratégia para a formação de um profissional generalista, humanista, ético, crítico e reflexivo, capaz de atuar em todos os níveis de atenção à saúde ${ }^{4}$.

Apesar da implementação de modificações nas estruturas curriculares e projetos pedagógicos dos cursos, visando incorporar as normas das DCN, ainda existem fragilidades a serem superadas. Estas podem estar fora do projeto pedagógico, como no gap existente entre o papel e a prática. Por exemplo, a responsabilidade da instituição formadora e corpo docente de formar profissionais críticos e reflexivos, que estejam aptos a buscar novos conhecimentos, porque estes vão surgir, sempre ${ }^{5}$.

É importante identificar a percepção dos graduandos frente a esse momento de transição para melhor compreender a problemática vocacional e investigar pontos relevantes a serem considerados na formulação de propostas de intervenção com esta população. Dessa forma, objetivou-se com este estudo avaliar a autopercepção do estudante de odontologia quanto às habilidades adquiridas na graduação para o início de suas atividades profissionais, além de identificar aspectos de seu projeto profissional.

\section{METODOLOGIA}

Trata-se de uma pesquisa transversal, exploratória, em que participaram estudantes de Odontologia de duas Instituições de Ensino Superior brasileiras, uma pública (Região Sudeste) e uma privada (Região Nordeste), selecionadas por conveniência. A instituição pública oferta 144 vagas e a privada 90 vagas, anualmente. Não houve a intenção de comparação entre as duas instituições. Duas escolas foram incluídas com o objetivo de enriquecer a diversidade dos contextos educacionais.

De acordo com documentos das duas instituições, a pública possui como missão "gerar e difundir conhecimentos científicos, tecnológicos e culturais, destacando-se como instituição de referência nacional, formando indivíduos críticos $e$ éticos, com uma sólida base científica e humanística, comprometidos com intervenções transformadoras na sociedade e com o desenvolvimento socioeconômico regional $e$ 
nacional", enquanto a privada visa "promover educação superior de qualidade com conhecimento crítico e socialmente comprometido".

A coleta de dados foi realizada por meio de questionário autoaplicável entre os meses de agosto e novembro de 2016. O pesquisador principal coletou todos os dados.

Foram incluídos no estudo estudantes regularmente matriculados no último período do curso, que aceitaram participar. Como os questionários foram aplicados em sala de aula, em ambas as instituições, os estudantes que não estavam presentes no momento da coleta de dados foram excluídos da pesquisa.

O questionário foi formulado em cinco partes: 1. Modalidade de inserção no mundo do trabalho (consultório próprio, clínica como empregado e serviço público); 2. Expectativas para os primeiros anos de formado quanto à continuidade de estudos (aperfeiçoamento, especialização, mestrado e doutorado); 3. Perguntas relacionadas às habilidades $\mathrm{e}$ competências previstas nas DCN para os cursos de Odontologia, a partir de uma lista organizada, onde se colocava como resposta para cada item da lista, "sim" ou "ainda não"; o primeiro bloco continha habilidades gerais para situações relacionadas à prática da Odontologia (liderança, gerenciamento, docência e realização de ações de educação, prevenção e promoção de saúde em nível coletivo) e o segundo, habilidades clinicas representadas pelos procedimentos odontológicos em todas as áreas (diagnóstico, restaurações, cirurgias e outros); 4. Questões relacionadas à pretensão salarial quando recém-formados e após 5 anos; 5 . Proficiência em idiomas (português, inglês, espanhol e outras línguas).

Para adequação do questionário, foi realizado um estudo piloto com 10 acadêmicos do nono período do Curso de Odontologia em cada uma das instituições com o objetivo de identificar as questões que geravam dúvidas. Após a análise, foi feita a alteração de um enunciado, no qual se verificou a necessidade de melhorar o entendimento da pergunta. Estes estudantes não foram incluídos na amostra final.

$\mathrm{Na}$ aplicação do questionário foi previamente explicado o objetivo do estudo a todos os entrevistados, e o termo de consentimento livre e esclarecido foi assinado por aqueles que concordaram em participar, observando-se as exigências éticas em pesquisa. Os dados foram analisados de forma descritiva por meio do programa estatístico SPSS versão 23.

Esta pesquisa foi aprovada pelo Comitê de Ética em Pesquisa institucional sob o parecer CAAE: 58974016.1.0000.5149.

\section{RESULTADOS}

Dos 132 questionários distribuídos 103 retornaram, com taxa de resposta de 78,03\%.

Quanto à pretensão de inserção no mercado de trabalho, a maioria dos estudantes pretende iniciar a vida profissional em clínica privada e/ou publica $(87 ; 84,46 \%)$, como prestador de serviços, com ou sem vínculo empregatício, modalidade claramente não liberal (tabela 1).

Os planos relacionados à continuidade dos estudos envolveram, principalmente, cursar aperfeiçoamento e/ou especialização $(72 ; 69,89 \%)$, coerente com a proposta de dedicação ao atendimento clínico (tabela 2).

A tabela 3 apresenta os resultados quanto à autopercepção dos estudantes à respeito de competências gerais para realização das atividades profissionais. Eles se sentem seguros para acolher e estabelecer vínculos com os pacientes e também para realizar atividades coletivas (promoção de saúde, prevenção e educação para a saúde). No entanto, percebeu-se insegurança quanto à realização de tarefas que envolvem questões como o gerenciamento de serviços públicos e privados, assumir cargos em entidades e liderança de equipe. 
O ser professor na área de saúde foi assinalado por 21,32\% dos estudantes, embora 31 (29,93\%) tenham colocado como proposta o mestrado e/ou doutorado em seus planos.

A maioria dos estudantes possui alta confiança para a realização de procedimentos de atenção primária. Foram apontadas dificuldades para a realização de endodontia de dentes multirradiculares, extração de dentes inclusos, prótese fixa e perícias odontológicas. Menos de 5\% dos entrevistados sentem-se confiantes para realizar implantes e prótese sobre implantes (tabela 4).

Tabela 1. Distribuição dos estudantes de Odontologia quanto à pretensão inicial de atuação profissional

\begin{tabular}{lcc}
\hline \hline Atuação profissional & $\mathrm{n}$ & $\%$ \\
\hline Clínica como empregado terceirizado & 37 & 35,92 \\
Clínica como empregado terceirizado e serviço público & 27 & 26,21 \\
Serviço público & 23 & 22,33 \\
Consultório próprio & 9 & 8,73 \\
Consultório próprio e serviço público & 4 & 3,88 \\
Consultório próprio, clínica como empregado terceirizado e serviço público & 2 & 1,94 \\
Consultório próprio e clínica como empregado terceirizado & 1 & 0,97 \\
\hline \hline
\end{tabular}

Tabela 2. Distribuição dos estudantes de Odontologia quanto à pretensão de continuidade de estudos

\begin{tabular}{lcc}
\hline \hline Continuidade de estudo & $\mathrm{n}$ & $\%$ \\
\hline Especialização & 26 & 25,24 \\
Aperfeiçoamento e especialização & 24 & 23,30 \\
Aperfeiçoamento & 22 & 21,35 \\
Mestrado & 7 & 6,79 \\
Especialização e mestrado & 7 & 6,79 \\
Aperfeiçoamento, especialização e mestrado & 5 & 4,85 \\
Aperfeiçoamento e mestrado & 4 & 3,88 \\
Nenhum & 3 & 2,77 \\
Mestrado e doutorado & 2 & 1,94 \\
Aperfeiçoamento, especialização e doutorado & 1 & 0,97 \\
Especialização, mestrado e doutorado & 1 & 0,97 \\
Aperfeiçoamento, especialização, mestrado e doutorado & 1 & 0,97 \\
\hline \hline
\end{tabular}

Com relação à autopercepção dos estudantes sobre proficiência em idiomas verificou-se que pelo menos $86,40 \%(\mathrm{n}=89)$ dos entrevistados afirmam que falam, leem ou escrevem bem português. Para a língua inglesa este índice apresentou-se entre $12,62 \%$ e $28,15 \%$, enquanto para a língua espanhola variou entre $8,73 \%$ e $18,44 \%$. Com relação a outros idiomas, $10,60 \%$ dos estudantes relataram conhecimento de outras línguas (francesa, japonesa, italiana e grega).

Quanto à remuneração, cerca de $50 \%$ dos estudantes apontaram expectativa de salário mensal médio entre $\mathrm{R} \$ 2.000,00$ e $\mathrm{R} \$ 3.000(2,27$ e 3,40 salários mínimos, SM) para o início da carreira, chegando a média de valores entre $\mathrm{R} \$ 6.000,00$ e $\mathrm{R} \$$ 8.000,00 (6,81 e 9,08 SM) 5 anos após a formatura, tempo que se considera minimamente necessário para a estabilidade financeira, ainda que relativa. 
Tabela 3. Distribuição dos estudantes de Odontologia quanto à autopercepção para realização de habilidades gerais adquiridas durante a formação acadêmica

\begin{tabular}{lcc}
\hline Habilidades gerais & $\mathrm{n}$ & $\%$ \\
\hline Acolhimento do paciente & 101 & 98,05 \\
Construção de vínculo com o paciente & 99 & 96,11 \\
Realizar ações coletivas de promoção de saúde & 97 & 94,17 \\
Ter iniciativa & 95 & 92,23 \\
Realizar ações coletivas de prevenção & 95 & 92,23 \\
Realizar ações coletivas de educação em saúde & 94 & 91,26 \\
Ser empregador & 78 & 75,72 \\
Gerenciar um consultório & 44 & 42,71 \\
Liderar equipe de saúde & 35 & 33,98 \\
Gerenciar serviços públicos de saúde & 33 & 32,03 \\
Assumir cargos em entidades de classe & 32 & 31,06 \\
Gerenciar serviços privados de saúde & 29 & 28,15 \\
Ser professor na área de saúde & 22 & 21,35 \\
\hline \hline
\end{tabular}

Tabela 4. Distribuição dos estudantes de Odontologia quanto à autopercepção de competências adquiridas durante a formação acadêmica

\begin{tabular}{lcc}
\hline \hline \multicolumn{1}{c}{ Procedimentos clínicos } & $\mathrm{n}$ & $\%$ \\
\hline Diagnóstico de carie & 103 & 100,00 \\
Atuar como clínico & 102 & 99,02 \\
Anestesia & 101 & 98,05 \\
Diagnóstico de doença periodontal & 100 & 97,08 \\
Raspagem supra gengival & 99 & 96,14 \\
Prescrição de analgésicos & 98 & 95,14 \\
Polimento coronário & 97 & 94,17 \\
Endodontia de unirradiculares & 96 & 93,26 \\
Raspagem sub gengival & 96 & 93,26 \\
Restaurações em amalgama & 94 & 91,26 \\
Prescrição de anti-inflamatórios & 94 & 91,26 \\
Diagnóstico de defeitos do esmalte & 93 & 90,29 \\
Restaurações estéticas & 91 & 88,34 \\
Atendimento de crianças & 88 & 85,43 \\
Extração de restos radiculares & 86 & 83,49 \\
Diagnóstico de oclusopatias & 84 & 81,55 \\
Prescrição de antibióticos & 78 & 75,72 \\
Pequena cirurgia periodontal & 74 & 71,84 \\
Restaurações indiretas & 72 & 69,90 \\
Diagnóstico de lesões de mucosa & 71 & 68,93 \\
Prótese total removível & 64 & 62,13 \\
Coroas protéticas & 61 & 59,22 \\
Endodontia de decíduos & 58 & 56,31 \\
Prótese fixa & 45 & 43,68 \\
Prótese parcial removível & 43,68 \\
Ajuste oclusal & 45 & 40,77 \\
Atendimento de pacientes especiais & 42 & 40,77 \\
Endodontia de multirradiculares & 42 & 35,92 \\
Extração de dentes inclusos & 37 & 35,92 \\
Pericias odontológicas & 37,59 \\
Prótese sobre implantes & 14 & 4,85 \\
Implantes & 5 & 1,88 \\
\hline \hline
\end{tabular}




\section{DISCUSSÃO}

É frequente observar, na prática docente, a ansiedade dos estudantes com o que consideram necessário aprender, na sua percepção, muitas vezes desproporcional ao tempo de curso. Consideram sua formação insuficiente para os desafios do mundo do trabalho ${ }^{6}$.

Por isto a ideia deste estudo. Pretendeu-se verificar a segurança autopercebida entre estudantes concluintes em dois cursos de Odontologia, considerando aspectos relacionados à sua inserção no mundo do trabalho, originários das DCN.

Os estudantes mostraram conhecer a realidade que os espera. Somente nove dos 132 participantes colocaram como opção o consultório próprio. A inserção majoritária foi a prestação de serviço em setores privados e/ou públicos (tabela 1).

O trabalho de Zitzmann et al. ${ }^{7}$ realizado com egressos encontrou que $76,4 \%$ destes estavam alocados na rede privada. Apesar do contrato de trabalho na maioria das vezes não incluir o vínculo empregatício, eles se sentem empregados, com possibilidade de auferir renda mensal, considerada impossível na modalidade consultório próprio.

No entanto os cirurgiões-dentistas, ao elegerem esta condição como ideal a ser perseguido, o fazem de forma antagônica à grande parte dos trabalhadores, que procuram avidamente a inserção regida pelo regime salarial. O consentimento da subcondição trabalhista imposta pelo mercado estimula a precarização profissional na odontologia ${ }^{8}$. Mas eles identificam este problema por isto dividem esta possibilidade com a inserção na rede pública, combinada ou não com a rede privada.

Alguns autores encontraram preferência de inserção por meio de carreira pública ${ }^{9}$. Exemplo disso é uma pesquisa realizada em um curso de Odontologia no Estado de Minas Gerais, Brasil, na qual foi identificado maior percentual $(89,0 \%)$ de estudantes de todos os semestres com intenção de início de carreira no serviço público ${ }^{10}$. Evidenciase, portanto, o distanciamento do início de carreira em consultório particular próprio e o desejo dos estudantes por oportunidades de carreira que ofereçam assalariamento e alguma segurança financeira.

No que concerne aos planos de continuidade de estudos, apenas $2,77 \%$ da amostra não pretende cursar pós-graduação. Há um alto índice de estudantes que almejam especializar-se: 63,09\% pretendem cursar especialização, e desses, $37,85 \%$ pretendem cursar especialização e algum outro curso como aperfeiçoamento, mestrado e doutorado (tabela 2).

Esta tendência é consoante a outros estudos ${ }^{11,12}$. Este dado sugere a tendência à calcificação do modelo biomédico de atenção à saúde, que enfatiza a especialização ao invés da abordagem generalista ${ }^{13}$. Diante deste cenário, é possível interpelar se esta escolha é consciente ou não, já que a estrutura curricular dos cursos de odontologia é composta de intensa prática clínica $^{14,15}$.

Por outro lado, é preciso considerar que não existe certeza da suficiência da formação frente ao mundo do trabalho ou mesmo quanto a alguns procedimentos clínicos. Este é um problema que, segundo os estudantes, a especialização e $\mathrm{o}$ aperfeiçoamento poderiam resolver ${ }^{6}$.

Quanto às habilidades gerais necessárias ao exercício da profissão, menos de $43 \%$ da amostra demonstrou aptidão para tarefas envolvendo gerenciamento, liderança de equipe de saúde e assumir cargo em entidade de classe, enquanto mais de $75 \%$ apontou confiança para a realização de ações coletivas, ser empregador, ter iniciativa, acolhimento e construção de vínculo com o paciente, o que revela certa contradição pois estas últimas funções envolvem a capacidade de ser líder. ${ }^{4}$.

Os índices aqui encontrados mostram baixo 
percentual de estudantes com pretensão de seguir carreira como docentes e pesquisadores. Este dado, somado ao desejo de seguir estudos em cursos de especialização, enfatiza a tendência na busca pela atuação clínica. Diversamente, uma pesquisa com egressos uma universidade da Suíça mostrou que mais de $80 \%$ dos graduados havia completado ou estava cursando doutorado ${ }^{7}$. É imprescindível o incentivo à pós-graduação stricto senso no Brasil, lembrando que existem hoje os mestrados e doutorados profissionais, que pretendem esta formação voltada para o trabalho não docente.

Com relação às tarefas administrativas e ao empreendedorismo, as DCN procuram nortear a formação para o desenvolvimento de habilidades como a liderança, a administração, o gerenciamento e a comunicação. Nesta pesquisa os resultados mostraram insegurança dos estudantes nestas competências, dados semelhantes aos encontrados em um trabalho com estudantes de todos os semestres de um curso de Odontologia no qual a maioria dos entrevistados julgou-se incapaz de atividades como conduzir reuniões administrativas e liderar a equipe odontológica ${ }^{16}$.

Os estudantes transparecem uma compreensão limitada destas habilidades. Observase que somente $33,98 \%$ relataram estar aptos a liderar equipes profissionais. No entanto, $75,72 \%$ considerou que estaria em condições de ser empregador. Ao mesmo tempo, gerenciar serviços de saúde privados foi percebida como habilidade pouco apreendida $(28,15 \%)$.

Outro aspecto a ser considerado é a opção pelo serviço público. Atualmente, capitaneado pela Estratégia Saúde da Família, o serviço público trabalha com base em equipes e, fatalmente, estas habilidades consideradas fracas pelos estudantes serão necessárias para que o trabalho flua e se estabeleçam relacionamentos proveitosos ${ }^{17}$.

Uma pesquisa na qual se promoveu a implementação de atividades relacionadas à recepção do paciente como o agendamento de consultas, o acolhimento e liberação do paciente na recepção, o preparo de faturamento e a emissão de documentos, comprovou a melhoria das habilidades administrativas de estudantes de Odontologia ${ }^{18}$.

Leadbeatter et $a l .{ }^{16}$ conceituam essas habilidades complementares como a "supercomplexidade da prática odontológica contemporânea", ressaltando que o aprendizado para a prática odontológica atual não pode ser realizado apenas pela aquisição de conhecimentos teóricos e habilidades técnicas, mas os estudantes precisam estar preparados para situações sem solução óbvia e perguntas sem resposta direta. Da mesma forma, outros autores ressaltam a importância de que o currículo educacional odontológico destaque atividades como gestão empresarial, gestão do estresse e habilidades de comunicação ${ }^{19}$.

Com relação às habilidades para procedimentos clínicos, observou-se que os estudantes se sentem confiantes para a maioria daqueles contemplados pelas DCN, demostrando menores índices de segurança para a realização de procedimentos de maior complexidade tecnológica ou especializados, como próteses, endodontia de dentes multirradiculares, perícias odontológicas e implantes odontológicos. Um estudo com resultados similares sugere que o aumento da atividade clínica em procedimentos complexos pode melhorar a autoconfiança dos estudantes ${ }^{20}$. No entanto, algumas destas habilidades são próprias de prática especializada. Frente aos dados, pode-se inferir que a formação para a clínica geral parece estar sendo implementada.

Algumas das habilidades clínicas fazem parte do rol de necessidades e demandas, sobretudo da população adulta e idosa, como próteses unitárias (restaurações indiretas e coroas protéticas), próteses removíveis ou tratamentos endodônticos em decíduos. Estas são da competência do nível secundário de atenção à 
saúde, mas exigência do trabalho diário do clínico no setor privado. Deste modo justifica-se mais uma vez a intenção do aperfeiçoamento ou especialização.

Preocupa o fato da dificuldade no diagnóstico de lesões de mucosa. É preciso que seja claro para os estudantes que somente a experiência e a lida diária formam o estomatologista. Mas o exame, a identificação ou o encaminhamento quando em presença de qualquer anormalidade, é uma habilidade fundamental a ser adquirida.

Nota-se paradoxo encontrado com relação à realização de restaurações, com índice de confiança entre $69,90 \%$ e $91,26 \%$, e ajuste oclusal, $40,77 \%$, que são procedimentos diretamente relacionados, mas que podem estar sendo fracionados na organização do ensino. Trabalhos futuros podem melhor investigar esses pontos contraditórios.

Os dados encontrados quanto à proficiência em idiomas revelam dificuldade dos estudantes para comunicação em línguas estrangeiras, o que pode representar potencial barreira frente ao processo de atualização científica ou internacionalização da Odontologia ${ }^{21-23}$. Embora não seja a língua oficial da maioria dos países europeus e latino-americanos, a língua inglesa é comum ao intercâmbio científico e cultural, sendo, portanto, importante como segundo idioma. Além disso grande parte da literatura é produzida na língua inglesa. Esta dificuldade pode, portanto, tornar-se um empecilho à atualização, necessária e imprescindível.

A maioria dos estudantes de Odontologia em todo o mundo não possuem nenhum núcleo de formação em inglês. $\mathrm{Na}$ Arábia Saudita, pesquisadores constataram a necessidade de maior foco ao ensino da língua inglesa na educação préuniversitária de estudantes de Odontologia a fim de melhor prepará-los aos desafios da educação universitária ${ }^{24}$. Da mesma forma, com o objetivo de aprofundar o conhecimento das motivações de estudantes japoneses e fornecer informações a respeito da internacionalização da Odontologia, estudos concluíram que são necessários incentivos e estratégias motivacionais para formar clínicos, educadores e pesquisadores atuantes em um mundo sem fronteiras ${ }^{22,23}$

Quanto à expectativa de remuneração, os estudantes apontaram o salário mensal médio entre $\mathrm{R} \$ 2.000,00$ e $\mathrm{R} \$ 3.000$ (2,27 e 3,40 SM) para o início da carreira, chegando a média de valores entre $\mathrm{R} \$ 6.000,00$ e $\mathrm{R} \$$ 8.000,00 (6,81 e 9,08 SM) aos cinco anos após formatura ${ }^{25}$. Isso aponta para expectativas menos irreais dos estudantes a respeito do mercado de trabalho, já que de acordo com levantamento do Instituto de Pesquisa Econômica Aplicada ${ }^{26}$, divulgado em 2013, a renda dos dentistas brasileiros varia de acordo com a região do país, sendo a remuneração média de $\mathrm{R} \$$ $5.367,00$ (7,91 SM) mensais para todos os níveis de experiência e atividade.

Estudos com o intuito de avaliar e compreender as percepções de estudantes em relação ao mercado de trabalho, habilidades e competências adquiridas ao longo da graduação são importantes para a melhoria das atividades educacionais.

Pode-se identificar nesta pesquisa algumas mudanças e tendências que transcorrem na Odontologia, com relação à inserção no mundo do trabalho e aos ganhos possíveis, estando bem próximos da realidade. Pretendem ser clínicos e isto os impele a procurar se aperfeiçoar ou especializar, decorrente possivelmente de algumas habilidades em áreas clínicas, nas quais não se sentem seguros para atuar. Mas algumas habilidades gerais, também insuficientes ou não adquiridas, não parecem ser preocupação ainda, uma vez que não planejaram ainda como resolvêlas.

Torna-se necessário identificar porque a área clínica, com a maior carga horária em cursos de graduação, ainda se constitui em maior reforço à insegurança percebida pelos estudantes 
concluintes, prestes a se inserir no mundo do trabalho.

Sugere-se a tentativa de novas maneiras de ensinagem, mais reflexivas e menos fechadas em protocolos, dando espaço para a criatividade e para a reinvenção. A construção e não a repetição do conhecimento pode ser a saída.

As DCN existem, no papel, e há 15 anos estão vigorando, mas o progresso na área de habilidades e competências ainda é pequeno. Precisamos dar o salto de qualidade na formação profissional. Não é o aumento da carga horária, como pensam os estudantes, que trará o sucesso. Não é aumentando horas com o professor. É preciso capacitar os futuros profissionais a gerenciar sua própria vida, seu aprendizado. Com a autonomia presente, a insegurança desaparece.

Entre as limitações deste estudo destaca-se a amostra não probabilística e não calculada em relação ao número total de estudantes concluintes dos cursos de Odontologia no Brasil. Além disso, utilizou instrumentos autoaplicáveis e os indivíduos podem não responder com rigor a essas ferramentas de pesquisa. Contudo, este trabalho mostra importantes indicativos a respeito da formação do acadêmico de Odontologia, acrescentando à literatura algumas questões que até então não haviam sido levantadas. Sugere-se que futuramente sejam utilizados questionários validados a fim de produzir resultados com maior grau de confiança.

\section{CONCLUSÃO}

Os estudantes concluintes de curso de Odontologia participantes deste estudo compreendem bem a realidade do mundo do trabalho, já que percebem sua inserção como prestadores de serviços ou empregados, com ganhos compatíveis com a realidade; apresentamse confiantes na aquisição de algumas habilidades gerais, mas se sentem insuficientes nas questões ligadas ao gerenciamento e liderança. Apresentam- se confiantes na aquisição de algumas habilidades clínicas, sobretudo as relacionadas às atividades da Atenção Primária em Saúde. A intenção da continuidade dos estudos é provocada não pela necessidade do aprendizado continuado, mas pela percepção de incompletude do ensino. Não se consideram fluentes em outras línguas, condição importante para o desenvolvimento profissional.

\section{ABSTRACT \\ Inclusion in the dental job market: perception of dental undergraduate students about skills acquired and expectations}

The aims of the present study were to evaluate the perceptions of dental students regarding the skills acquired during their undergraduate course to begin their professional activities and identify aspects of the professional plans. Student's from the tenth semester of two higher education institutions answered a self-administrated questionnaire addressing the skills stipulated in the Brazilian National Dental Curriculum Guidelines and their plans for beginning their professional careers. One hundred three of the 132 questionnaires were answered. Most students $(\mathrm{n}=87 ; 84.46 \%)$ planned to begin their careers in a private and/or public clinic as a service provider and planned to continue their studies in specialization and/or improvement courses $(\mathrm{n}=72 ; 69.89 \%)$. Most students felt confident regarding clinical skills related to basic care procedures $(>70 \%)$, but much less confident regarding specialized procedures. With regard to general skills, the students reported difficulties in terms of leadership and management (42.71\%). They are not fluent in any foreign languages $(28.15 \%)$ and have realistic expectations of financial gains. They understand the job market well, perceive the need to continue their studies, are confident with regard to some acquired general and clinical skills and perceive that others are insufficient. Reconsidering the mode of teaching, the teaching-learning process and evaluation methods could be a way to potentiate the National Dental Curriculum Guidelines.

Descriptors: Dental Students. Professional Competence. Teaching. Health Care Sector. 
RESUMEN

Inclusión en el mercado de trabajo de odontología: percepción de estudiantes de pregrado sobre habilidades adquiridas y expectativas

Esta investigación tuvo como objetivo evaluar la autopercepción del estudiante de odontología cuanto a las habilidades adquiridas en la graduación para el inicio de sus actividades profesionales, además de identificar aspectos de su proyecto profesional. Los estudiantes del décimo período de dos Instituciones de Enseñanza Superior contestaran a un cuestionario auto aplicable, con preguntas relacionadas con las habilidades y competencias previstas en las Directrices Curriculares Nacionales de Odontología, y relativas a su proyecto inicial de vida profesional. De los 132 cuestionarios distribuidos, 103 fueron contestados. La mayoría de los estudiantes consideró iniciar carrera en clínica privada y/o publica $(87 ; 84,46 \%)$, como proveedor de servicios. Acerca del plan de los estudiantes para la continuidad de estudios, planean cursos de especializacion y/o perfeccionamento (72; $69,89 \%$ ). Tienen seguridad en habilidades clínicas relacionadas con procedimientos de Atención Básica (> 70\%) y mucho menos en procedimientos especializados. En cuanto a las habilidades generales presentan dificultades en acciones de liderazgo y gestión $(<42,71)$. No tiene buena convivencia con lengua extranjera (28,15\%), presentan expectativas de ganancias financieras cercanas a la realidad. Comprenden bien la realidad del mundo del trabajo, perciben la necesidad de continuar los estudios, están seguros de algunas habilidades generales y clínicas adquiridas y perciben que algunas son insuficientes. Rever el modo de enseñar, la relación enseñanzaaprendizaje y la metodología de evaluación puede ser un camino para hacer vivas las Directrices Nacionales Curriculares para la Odontología.

Descriptores: Estudiantes de Odontologia. Competencia Professional. Enseñanza. Sector de Atención de Salud.

\section{REFERÊNCIAS}

1. Barros JAC. Pensando o processo saúde-doença: a que responde o modelo biomédico? Saúde Soc.
2002;11(1): 67-84.

2. Cruvinel VRN, Franco EJ, Bezerra L, Alves MM, Miranda AF, Carvalho DR. A formação do cirurgião-dentista generalista na Universidade Católica de Brasília. Rev ABENO. 2010; 10(2):5-11.

3. Ferreira NP, Ferreira AP, Freire MCM. Mercado de trabalho na Odontologia: contextualização e perspectivas. Rev Odontol UNESP. 2013; 42(4):304-9.

4. Brasil. Ministério da Educação. Conselho Nacional de Educação. Câmara de Educação Superior. Diretrizes Curriculares Nacionais para o Curso de Graduação em Odontologia. Diário Oficial da União, 1: 10, 2002.

5. Souza FA, Paiano M. Desafios e dificuldades enfrentadas pelos profissionais de enfermagem em início de carreira. REME Rev Min Enferm. 2011;15(2):267-73.

6. Gondim SMG. Perfil profissional e mercado de trabalho: relação com a formação acadêmica pela perspectiva de estudantes universitários. Estud Psicol. 2002; 7(2): 299-309.

7. Zitzmann NU, Yoon-Buchel N, Buhler J, Dettwiler CA, Weiger R. Evaluation of the dental curriculum at the University of Basel - Does the Master of dental medicine adequately prepare for the professional practice? Swiss Dent J SSO. 2016;126(12): 1134-9.

8. Bleicher L. Precarização do trabalho do cirurgião-dentista na cidade de Salvador, Bahia. Rev Baiana Saúde Pública. 2012; 6(3):668-82.

9. Halawany HS, Binassfour AS, AlHassan WK, Alhejaily RA, Maflehi NA, Jacob V, et al. Dental specialty, career preferences and their influencing factors among final year dental students in Saudi Arabia. Saudi Dent J. 2017; 29(1):15-23.

10. Costa SM, Silveira MF, Durães SJA, Abreu MHNG, Bonan PRF. Perceptions of dental students regarding dentistry, the job Market and the public healthcare system. Ciênc Saúde Colet. 
2012;17(5):1285-96.

11. Che Musa MF, Bernabe E, Gallagher JE. Career expectations and influences among dental students in Malaysia. Int Dent J. 2016;66(4):229-36.

12. Matos MS, Tenório RM. Expectativas de estudantes de Odontologia sobre o campo de trabalho odontológico e o exercício profissional. Rev Bras Pesqui Saúde. 2011; 13(4):10-21.

13. Whitney EM, Walton JN, Aleksejuniene J, Schonwetter DJ. Graduating dental students' views of competency statements: importance, confidence, and time trends from 2008 to 2012. J Dent Educ. 2015;79(3):322-30.

14. Brasil. Ministério da Educação. Conselho Nacional de Educação. Câmara de Educação Superior. Resolução n²/2007. Dispõe sobre carga horária mínima e procedimentos relativos à integralização e duração dos cursos de graduação, bacharelados, na modalidade presencial. Diário Oficial da União, Brasília: DF, 2007

15. Honey J, Lynch CD, Burke FM, Gilmour ASM. Ready for practice? A study of confidence levels of final year dental students at Cardiff University and University College Cork. Eur J Dent Educ. 2011;15(2):98-103.

16. Leadbeatter D, Peck C. Are dental students ready for supercomplex dental practice? Eur J Dent Educ. 2018;22(1):e116-e121.

17. Lichtenstein N, Ensmann I, Haak R, Hallal H, Kupke J, Matthes J, et al. "May I help you?" Evaluation of the new student service at the reception desk during the clinical courses at the Department of Operative Dentistry and Periodontology as a part of a longitudinal curriculum of social and communicative competences for dental students. GMS Z Med Ausbild. 2015;32(3):1-18.

18. Pereira RCA, Rivera FJU, Artmann E. The multidisciplinary work in the family health strategy: a study on ways of teams. Interface (Botucatu). 2013;17(45): 327-40

19. Badger GR, Fryer CES, Giannini PJ, Townsend
JA, Huja S. Helping dental students make informed decisions about private practice employment options in a changing landscape. $\mathbf{J}$ Dent Educ. 2015;79(12):1396-401.

20. Divaris K, Barlow PJ, Chendea SA, Cheong WS, Dounis A, Dragan IF, et al. The academic environment: the students'perspective. Eur J Dent Educ. 2008;12(1):120-30.

21. Morse Z, Nakahara S. English language education in Japanese dental schools. Eur J Dent Educ. 2001; 5(4):168-72.

22. Takehara S, Wright FAC, Kawaguchi Y, Ishida Y, Morio I, Tagami J. Characteristics of undergraduate dental students in Japan: English competency and willingness to study abroad. Int Dent J. 2016;66(5):311-7.

23. Takehara S, Wright FAC, Kawaguchi Y, Ishida Y, Morio I, Tagami J. Characteristics of undergraduate dental students in Japan: english competency and willingness to study abroad. Int Dent J. 2016;66(5):311-7.

24. El Tantawi M, Al-Ansari A, Sadaf S, AlHumaid J. Evaluating the English language scientific writing skills of Saudi dental students at entry level. EMHJ. 2016; 22(2): 148-53.

25. Matos MS, Tenório RM. Expectativas de estudantes de Odontologia sobre o campo de trabalho odontológico e o exercício profissional. Rev Bras Pesqui Saúde. 2011; 13(4):10-21.

26. Brasil. Secretaria de Assuntos Estratégicos. Instituto de Pesquisa Econômica Aplicada. Diretoria de Estudos e Políticas Setoriais, de Inovação, Regulação e Infraestrutura. Radar: tecnologia, produção e comércio exterior. Brasília: Ipea, 2013.

\section{Correspondência para:}

Taiomara Vieira Mania

e-mail: taiomaravieiramania@hotmail.com

Faculdade Independente do Nordeste, Departamento de Odontologia

Av. Luis Eduardo Magalhães, 1305, Candeias

45.055-030 Vitória da Conquista/BA 